\title{
Doubly Warped Products in Locally Conformal Almost Cosymplectic Manifolds
}

\author{
Andreea Olteanu*
}

(Communicated by Ion Mihai)

\begin{abstract}
Recently, the author established a general inequality for doubly warped products in arbitrary Riemannian manifolds [16]. In the present paper, we obtain similar inequalities for doubly warped products isometrically immersed in locally conformal almost cosymplectic manifolds. Some applications are derived.
\end{abstract}

Keywords: Doubly warped product; minimal immersion; totally real submanifold; locally conformal almost cosymplectic manifold.

AMS Subject Classification (2010): Primary: 53C40 ; Secondary: 53C25; 53B25; 53C42.

\section{Introduction}

Bishop and O'Neill [1] introduced the concept of warped products to study manifolds of negative sectional curvature. O'Neill discussed warped products and explored curvature formulas of warped products in terms of curvatures of components of warped products.

Doubly warped products can be considered as a generalization of singly warped products.

Let $\left(M_{1}, g_{1}\right)$ and $\left(M_{2}, g_{2}\right)$ be two Riemannian manifolds and let $f_{1}: M_{1} \rightarrow(0, \infty)$ and $f_{2}: M_{2} \rightarrow(0, \infty)$ be differentiable functions.

The doubly warped product $M=f_{f_{2}} M_{1} \times_{f_{1}} M_{2}$ is the product manifold $M_{1} \times M_{2}$ endowed with the metric

$$
g=f_{2}^{2} g_{1}+f_{1}^{2} g_{2} .
$$

More precisely, if $\pi_{1}: M_{1} \times M_{2} \rightarrow M_{1}$ and $\pi_{2}: M_{1} \times M_{2} \rightarrow M_{2}$ are natural projections, the metric $g$ is defined by

$$
g=\left(f_{2} \circ \pi_{2}\right)^{2} \pi_{1}^{*} g_{1}+\left(f_{1} \circ \pi_{1}\right)^{2} \pi_{2}^{*} g_{2} .
$$

The functions $f_{1}$ and $f_{2}$ are called warping functions. If either $f_{1} \equiv 1$ or $f_{2} \equiv 1$, but not both, then we obtain a warped product. If both $f_{1} \equiv 1$ and $f_{2} \equiv 1$, then we have a Riemannian product manifold. If neither $f_{1}$ nor $f_{2}$ is constant, then we have a non-trivial doubly warped product [20].

Let $x: f_{2} M_{1} \times_{f_{1}} M_{2} \rightarrow \widetilde{M}$ be an isometric immersion of a doubly warped product ${ }_{f_{2}} M_{1} \times_{f_{1}} M_{2}$ into a Riemannian manifold $\widetilde{M}$. We denote by $h$ the second fundamental form of $x$ and by $H_{i}=\frac{1}{n_{i}}$ trace $h_{i}$ the partial mean curvatures, where trace $h_{i}$ is the trace of $h$ restricted to $M_{i}$ and $n_{i}=\operatorname{dim} M_{i}(i=1,2)$.

The immersion $x$ is said to be mixed totally geodesic if $h(X, Z)=0$, for any vector fields $X$ and $Z$ tangent to $D_{1}$ and $D_{2}$, respectively, where $\mathrm{D}_{i}$ are the distributions obtained from the vectors tangent to $M_{i}$ (or more precisely, vectors tangent to the horizontal lifts of $M_{i}$ ).

In [4], B. Y. Chen studied the relationship between the Laplacian of the warping function $f$ and the squared mean curvature of a warped product $M_{1} \times_{f} M_{2}$ isometrically immersed in a Riemannian manifold $\widetilde{M}(c)$ of constant sectional curvature $c$ given by

$$
\frac{\Delta f}{f} \leq \frac{n^{2}}{4 n_{2}}\|H\|^{2}+n_{1} c
$$

where $n_{i}=\operatorname{dim} M_{i}, i=1,2$, and $\Delta$ is the Laplacian operator of $M_{1}$. Moreover, the equality case of (1.1) holds if and only if $x$ is a mixed totally geodesic immersion and $n_{1} H_{1}=n_{2} H_{2}$, where $H_{i}, i=1,2$, are the partial mean curvature vectors. 
Later, several Chen inequalities have been obtained for different submanifolds in ambient manifolds possessing different kind of structures (see [7], [8], [10], [11], [13], [14]).

In 2008, B. Y. Chen and F. Dillen extended inequality (1.1) to multiply warped product manifolds in arbitrary Riemannian manifolds (see [5]).

In [16], the present author established the following general inequality for arbitrary isometric immersions of doubly warped product manifolds in arbitrary Riemannian manifolds:

Theorem 1.1. Let $x$ be an isometric immersion of an n-dimensional doubly warped product $M=f_{f_{2}} M_{1} \times_{f_{1}} M_{2}$ into an m-dimensional arbitrary Riemannian manifold $\widetilde{M}^{m}$. Then:

$$
n_{2} \frac{\Delta_{1} f_{1}}{f_{1}}+n_{1} \frac{\Delta_{2} f_{2}}{f_{2}} \leq \frac{n^{2}}{4}\|H\|^{2}+n_{1} n_{2} \max \widetilde{K}
$$

where $n_{i}=\operatorname{dim} M_{i}, n=n_{1}+n_{2}, \Delta_{i}$ is the Laplacian operator of $M_{i}, i=1,2$ and $\max \widetilde{K}(p)$ denotes the maximum of the sectional curvature function of $\widetilde{M}^{m}$ restricted to 2-plane sections of the tangent space $T_{p} M$ of $M$ at each point $p$ in M. Moreover, the equality case of (1.2) holds if and only if the following two statements hold:

1. $x$ is a mixed totally geodesic immersion satisfying $n_{1} H_{1}=n_{2} H_{2}$, where $H_{i}, i=1,2$, are the partial mean curvature vectors of $M_{i}$.

2. at each point $p=\left(p_{1}, p_{2}\right) \in M$, the sectional curvature function $\widetilde{K}$ of $\widetilde{M}^{m}$ satisfies $\widetilde{K}(u, v)=\max \widetilde{K}(p)$ for each unit vector $u \in T_{p_{1}} M_{1}$ and each unit vector $v \in T_{p_{2}} M_{2}$.

Later, in [18] and [19], the present author studied doubly warped product submanifolds in generalized Sasakian space forms and $S$-space forms, respectively. In [6], M. Crasmareanu introduced and studied biwarped 3-metrics from the point of view of Webster curvature. In [21], D. W.Yoon, K. S. Cho, S. G. Han investigated warped product submanifolds in locally conformal almost cosymplectic manifolds.

Motivated by the studies of the above authors, the aim of this paper is to obtain similar inequalities for doubly warped products in locally conformal almost cosymplectic manifolds of pointwise constant $\varphi$-sectional curvature $c$.

\section{Preliminaries}

In this section, we recall some general definitions and basic formulas which will be used later. For more background on locally conformal almost cosymplectic manifolds, we recommend the reference [15].

Let $\widetilde{M}$ be a $(2 m+1)$-dimensional almost contact manifold (see [2]). Denote by $(\varphi, \xi, \eta)$ the almost contact structure of $\widetilde{M}$. Thus, $\varphi$ is a $(1,1)$-tensor field, $\xi$ is a vector field and $\eta$ a 1-form on $\widetilde{M}$ such that

$$
\varphi^{2}=-I+\eta \otimes \xi, \eta(\xi)=1 .
$$

Define an almost complex structure $J$ on the product manifold $\widetilde{M} \times \mathbb{R}$ by

$$
J\left(X, \lambda \frac{d}{d s}\right)=\left(\varphi X-\lambda \xi, \eta(X) \frac{d}{d s}\right),
$$

where $X$ and $\lambda \frac{d}{d s}$ are vectors tangent to $\widetilde{M}$ and $\mathbb{R}$, respectively, $\mathbb{R}$ being the real line with coordinate $s$.

The manifold $\widetilde{M}$ is said to be normal ([2]) if the almost complex structure $J$ is integrable (i.e., $J$ arises from a complex structure on $\widetilde{M} \times \mathbb{R}$ ). The necessary and sufficient condition for $\widetilde{M}$ to be normal is

$$
[\varphi, \varphi]+2 d \eta \otimes \xi=0,
$$

where $[\varphi, \varphi]$ is the Nijenhuis tensor of $\varphi$ defined by

$$
[\varphi, \varphi](X, Y)=[\varphi X, \varphi Y]-\varphi[\varphi X, Y]-\varphi[X, \varphi Y]+\varphi^{2}[X, Y]
$$

for any vector fields $X$ and $Y$ tangent to $\widetilde{M},[\cdot, \cdot]$ being the Lie bracket of vector fields.

Let $g$ be a Riemannian metric on $\widetilde{M}$ compatible with $(\varphi, \xi, \eta)$, such that

$$
g(\varphi X, \varphi Y)=g(X, Y)-\eta(X) \eta(Y)
$$


for any vector fields $X$ and $Y$ tangent to $\widetilde{M}$. Thus, the manifold $\widetilde{M}$ is almost contact metric and the quadruple $(\varphi, \xi, \eta, g)$ is its almost contact metric structure. Clearly, we have $\eta(X)=g(X, \xi)$ for any vector field $X$ tangent to $\widetilde{M}$. Let $\Phi$ denote the fundamental 2-form of $\widetilde{M}$ defined by $\Phi(X, Y)=g(\varphi X, Y)$ for any vector fields $X$ and $Y$ tangent to $\widetilde{M}$. The manifold $\widetilde{M}$ is said to be almost cosymplectic if the forms $\eta$ and $\Phi$ are closed, i.e., $d \eta=0$ and $d \Phi=0$, where $d$ is the operator of exterior differentiation. If $\widetilde{M}$ is almost cosymplectic and normal, then it is called cosymplectic ([2]). It is well known that the almost contact metric manifold is cosymplectic if and only if $\widetilde{\nabla} \varphi$ vanishes identically, where $\widetilde{\nabla}$ is the Levi-Civita connection on $\widetilde{M}$. The products of almost Kähler manifolds and $\mathbb{R}$ are the simplest examples of almost cosymplectic manifolds.

An almost contact metric manifold $\widetilde{M}$ is called a locally conformal almost cosymplectic manifold ([15]) if there exists a 1-form $\omega$ such that $d \Phi=2 \omega \wedge \Phi, d \eta=\omega \wedge \eta$ and $d \omega=0$. ([9])

A necessary and sufficient condition for a structure to be normal locally conformal almost cosymplectic is

$$
\left(\widetilde{\nabla}_{X} \varphi\right) Y=u(g(\varphi X, Y) \xi-\eta(Y) \varphi X),
$$

where $\omega=u \eta$. From $(2.2)$ it follows that $\widetilde{\nabla}_{X} \xi=u(X-\eta(X) \xi)$.

A plane section $\sigma$ in $T_{p} \widetilde{M}$ of an almost contact structure manifold $\widetilde{M}$ is called a $\varphi$-section if $\sigma \perp \xi$ and $\varphi(\sigma)=\sigma$. $\widetilde{M}$ is of pointwise constant $\varphi$ - sectional curvature if at each point $p \in \widetilde{M}$, the section curvature $\widetilde{K}(\sigma)$ does not depend on the choice of the $\varphi$ - section $\sigma$ of $T_{p} \widetilde{M}$ and, in this case for $p \in \widetilde{M}$ and for any $\varphi$ - section $\sigma$ of $T_{p} \widetilde{M}$, the function $c$ defined by $c(p)=\widetilde{K}(\sigma)$ is called the $\varphi$ - sectional curvature of $\widetilde{M}$. A locally conformal almost cosymplectic manifold $\widetilde{M}$ of dimension $\geq 5$ is of pointwise constant $\varphi$ - sectional curvature if and only if its curvature tensor $\widetilde{R}$ is of the form ([15])

$$
\begin{gathered}
\widetilde{R}(X, Y, Z, W)=\frac{c-3 u^{2}}{4}\{g(X, W) g(Y, Z)-g(X, Z) g(Y, W)\} \\
+\frac{c+u^{2}}{4}\{g(X, \varphi W) g(Y, \varphi Z)-g(X, \varphi Z) g(Y, \varphi W) \\
-2 g(X, \varphi Y) g(Z, \varphi W)\} \\
-\left(\frac{c+u^{2}}{4}+u^{\prime}\right)\{g(X, W) \eta(Y) \eta(Z)-g(X, Z) \eta(Y) \eta(W)\} \\
+g(Y, Z) \eta(X) \eta(W)-g(Y, W) \eta(X) \eta(Z)\} \\
+g(h(X, W), h(Y, Z))-g(h(X, Z), h(Y, W)),
\end{gathered}
$$

where $u$ is a function such that $\omega=u \eta, u^{\prime}=\xi u$ and $c$ is the pointwise constant $\varphi$ - sectional curvature of $\widetilde{M}$.

Let $M$ be an $n$-dimensional manifold isometrically immersed in a locally conformal almost cosymplectic manifold $\widetilde{M}$. Let $\nabla$ be the induced Levi-Civita connection of $M$. Then the Gauss and Weingarten formulas are given respectively by

$$
\begin{gathered}
\widetilde{\nabla}_{X} Y=\nabla_{X} Y+h(X, Y), \\
\widetilde{\nabla}_{X} V=-A_{V} X+D_{X} V
\end{gathered}
$$

for vector fields $X, Y$ tangent to $M$ and a vector field $V$ normal to $M$, where $h$ denotes the second fundamental form, $D$ the normal connection and $A_{V}$ the shape operator in the direction of $V$. The second fundamental form and the shape operator are related as

$$
g(h(X, Y), V)=g\left(A_{V} X, Y\right) .
$$

We also denote by $g$ the induced Riemannian metric on $M$ as well as on the locally conformal almost cosymplectic manifold $\widetilde{M}$.

Let $X$ be tangent to $M$ we put $\varphi X=P X+F X$, where $P X$ and $F X$ are the tangential and the normal components of $\varphi X$, respectively. The squared norm of $P$ for an othonormal basis $\left\{e_{1}, \ldots, e_{n}\right\}$ of $M$ is defined by

$$
\|P\|^{2}=\sum_{i, j=1}^{n} g^{2}\left(P e_{i}, e_{j}\right)
$$


and the mean curvature vector $H(p)$ at $p \in M$ is given by

$$
H=\frac{1}{n} \operatorname{traceh}=\frac{1}{n} \sum_{i=1}^{n} h\left(e_{i}, e_{i}\right) .
$$

As is known, $M$ is said to be minimal if $H$ vanishes identically.

Also, for any $r \in\{n+1, \ldots, 2 m+1\}$ we set

$$
h_{i j}^{r}=g\left(h\left(e_{i}, e_{j}\right), e_{r}\right), i, j \in\{1, \ldots, n\},
$$

and

$$
\|h\|^{2}=\sum_{i, j=1}^{n} g\left(h\left(e_{i}, e_{j}\right), h\left(e_{i}, e_{j}\right)\right)
$$

where $\left\{e_{n+1}, \ldots, e_{2 m+1}\right\}$ is an orthonormal basis of $T_{p}^{\perp} M$.

A submanifold $M$ is totally geodesic in $\widetilde{M}$ if $h=0$ and totally real submanifold if $P$ is identically zero, that is, $\varphi X \in T_{p}^{\perp} M$ for any $X \in T_{p} M, p \in M$.

For an $n$-dimensional Riemannian manifold $M$, we denote by $K(\pi)$ the sectional curvature of $M$ associated with a plane section $\pi \subset T_{p} M, p \in M$. For any orthonormal basis $e_{1}, \ldots, e_{n}$ of the tangent space $T_{p} M$, the scalar curvature $\tau$ at $p$ is defined by

$$
\tau(p)=\sum_{1 \leq i<j \leq n} K\left(e_{i} \wedge e_{j}\right) .
$$

Let $M$ be a Riemannian $p$-manifold and $\left\{e_{1}, \ldots, e_{p}\right\}$ be an orthonormal basis of $M$. For a differentiable function $f$ on $M$, the Laplacian $\Delta f$ of $f$ is defined by

$$
\Delta f=\sum_{j=1}^{p}\left\{\left(\nabla_{e_{j}} e_{j}\right) f-e_{j} e_{j} f\right\} .
$$

We recall the following general algebraic lemma of Chen for later use.

Lemma 2.1. [3] Let $n \geq 2$ and $a_{1}, a_{2}, \ldots, a_{n}, b$ real numbers such that

$$
\left(\sum_{i=1}^{n} a_{i}\right)^{2}=(n-1)\left(\sum_{i=1}^{n} a_{i}^{2}+b\right) .
$$

Then $2 a_{1} a_{2} \geq b$, with equality holding if and only if

$$
a_{1}+a_{2}=a_{3}=\ldots=a_{n} .
$$

\section{Doubly warped product submanifolds in locally conformal almost cosymplectic manifolds}

Next, we investigate doubly warped product submanifolds tangent to the structure vector field $\xi$ in a locally conformal almost cosymplectic manifold $\widetilde{M}(c)$.

Theorem 3.1. Let $x$ be an isometric immersion of an n-dimensional doubly warped product ${ }_{f_{2}} M_{1} \times_{f_{1}} M_{2}$ into a $(2 m+1)$-dimensional locally conformal almost cosymplectic manifold of pointwise constant $\varphi$-sectional curvature $c$ whose structure vector field $\xi$ is tangent to $M_{1}$. Then:

$$
n_{2} \frac{\Delta_{1} f_{1}}{f_{1}}+n_{1} \frac{\Delta_{2} f_{2}}{f_{2}} \leq \frac{n^{2}}{4}\|H\|^{2}+n_{1} n_{2} \frac{c-3 u^{2}}{4}-\left(\frac{c+u^{2}}{4}+u^{\prime}\right) n_{2}+\frac{3\left(c+u^{2}\right)}{4} n_{2},
$$

where $n_{i}=\operatorname{dim} M_{i}$ and $\Delta_{i}$ is the Laplacian operator of $M_{i}, i=1,2$. 
Proof. Suppose that $f_{2} M_{1} \times_{f_{1}} M_{2}$ is a doubly warped product submanifold of a locally conformal almost cosymplectic manifold $\widetilde{M}(c)$ with pointwise constant $\varphi$ - sectional curvature $c$ whose structure vector field $\xi$ is tangent to $M_{1}$. Since $f_{2} M_{1} \times_{f_{1}} M_{2}$ is a doubly warped product, then

$$
\left\{\begin{array}{c}
\nabla_{X} Y=\nabla_{X}^{1} Y-\frac{f_{2}^{2}}{f_{1}^{2}} g_{1}(X, Y) \nabla^{2}\left(\ln f_{2}\right), \\
\nabla_{X} Z=Z\left(\ln f_{2}\right) X+X\left(\ln f_{1}\right) Z
\end{array}\right.
$$

for any vector fields $X, Z$ tangent to $M_{1}$ and $M_{2}$, respectively, where $\nabla^{1}$ and $\nabla^{2}$ are the Levi-Civita connections of the Riemannian metrics $g_{1}$ and $g_{2}$, respectively. Here, $\nabla^{2}\left(\ln f_{2}\right)$ denotes the gradient of $\ln f_{2}$ with respect to the metric $g_{2}$.

If $X$ and $Z$ are unit vector fields, it follows that the sectional curvature $K(X \wedge Z)$ of the plane section spanned by $X$ and $Z$ is given by

$$
K(X \wedge Z)=\frac{1}{f_{1}}\left\{\left(\nabla_{X}^{1} X\right) f_{1}-X^{2} f_{1}\right\}+\frac{1}{f_{2}}\left\{\left(\nabla_{Z}^{2} Z\right) f_{2}-Z^{2} f_{2}\right\} .
$$

We choose a local ortonormal frame $\left\{e_{1}, \ldots, e_{n}, e_{n+1}, \ldots, e_{2 m+1}\right\}$ such that $e_{1}, \ldots, e_{n_{1}}=\xi$ are tangent to $M_{1}$, $e_{n_{1}+1}, \ldots, e_{n}$ are tangent to $M_{2}$ and $e_{n+1}$ is parallel to the mean curvature vectore $H$.

Then from (3.3), it follows that

$$
\sum_{\substack{1 \leq j \leq n_{1} \\ n_{1}+1 \leq s \leq n}} K\left(e_{j} \wedge e_{s}\right)=n_{2} \frac{\Delta_{1} f_{1}}{f_{1}}+n_{1} \frac{\Delta_{2} f_{2}}{f_{2}} .
$$

Using the equation of Gauss, we get

$$
n^{2}\|H\|^{2}=2 \tau+\|h\|^{2}-n(n-1) \frac{c-3 u^{2}}{4}-\frac{3\left(c+u^{2}\right)}{4}\|P\|^{2}+\left(\frac{c+u^{2}}{4}+u^{\prime}\right)(2 n-2),
$$

where $\tau$ denotes the scalar curvature of $f_{2} M_{1} \times_{f_{1}} M_{2}$, that is,

$$
\tau=\sum_{1 \leq i<j \leq n} K\left(e_{i} \wedge e_{j}\right)
$$

Let us consider that

$$
\delta=2 \tau-n(n-1) \frac{c-3 u^{2}}{4}-\frac{3\left(c+u^{2}\right)}{4}\|P\|^{2}+\left(\frac{c+u^{2}}{4}+u^{\prime}\right)(2 n-2)-\frac{n^{2}}{2}\|H\|^{2} .
$$

Then, (3.5) can be written as

$$
n^{2}\|H\|^{2}=2\left(\delta+\|h\|^{2}\right) .
$$

Thus with respect to the above orthonormal frame, the above equation takes the following form:

$$
\left(\sum_{i=1}^{n} h_{i i}^{n+1}\right)^{2}=2\left[\delta+\sum_{i=1}^{n}\left(h_{i i}^{n+1}\right)^{2}+\sum_{i \neq j}\left(h_{i j}^{n+1}\right)^{2}+\sum_{r=n+2}^{2 m+1} \sum_{i, j=1}^{n}\left(h_{i j}^{r}\right)^{2}\right] .
$$

Assume that $a_{1}=h_{11}^{n+1}, a_{2}=\sum_{i=2}^{n_{1}} h_{i i}^{n+1}$ and $a_{3}=\sum_{t=n_{1}+1}^{n} h_{t t}^{n+1}$. Then the above equation becomes

$$
\begin{aligned}
\left(\sum_{i=1}^{3} a_{i}\right)^{2} & =2\left[\delta+\sum_{i=1}^{3} a_{i}^{2}+\sum_{1 \leq i \neq j \leq n}\left(h_{i j}^{n+1}\right)^{2}+\sum_{r=n+2}^{2 m+1} \sum_{i, j=1}^{n}\left(h_{i j}^{r}\right)^{2}\right. \\
& \left.-\sum_{2 \leq j \neq k \leq n_{1}} h_{j j}^{n+1} h_{k k}^{n+1}-\sum_{n_{1}+1 \leq s \neq t \leq n} h_{s s}^{n+1} h_{t t}^{n+1}\right] .
\end{aligned}
$$

Thus $a_{1}, a_{2}, a_{3}$ satisfy the Lemma of Chen (for $n=3$ ), i.e.,

$$
\left(\sum_{i=1}^{3} a_{i}\right)^{2}=2\left(b+\sum_{i=1}^{3} a_{i}^{2}\right)
$$


with

$$
\begin{gathered}
b=\delta+\sum_{1 \leq i \neq j \leq n}\left(h_{i j}^{n+1}\right)^{2}+\sum_{r=n+2}^{2 m+1} \sum_{i, j=1}^{n}\left(h_{i j}^{r}\right)^{2}- \\
-\sum_{2 \leq j \neq k \leq n_{1}} h_{j j}^{n+1} h_{k k}^{n+1}-\sum_{n_{1}+1 \leq s \neq t \leq n} h_{s s}^{n+1} h_{t t}^{n+1} .
\end{gathered}
$$

Then $2 a_{1} a_{2} \geq b$, with equality holding if and only if $a_{1}+a_{2}=a_{3}$.

In the case under consideration, it follows that

$$
\begin{gathered}
\sum_{1 \leq j<k \leq n_{1}} h_{j j}^{n+1} h_{k k}^{n+1}+\sum_{n_{1}+1 \leq s<t \leq n} h_{s s}^{n+1} h_{t t}^{n+1} \geq \\
\geq \frac{\delta}{2}+\sum_{1 \leq \alpha<\beta \leq n}\left(h_{\alpha \beta}^{n+1}\right)^{2}+\frac{1}{2} \sum_{r=n+2}^{2 m+1} \sum_{\alpha, \beta=1}^{n}\left(h_{\alpha \beta}^{r}\right)^{2} .
\end{gathered}
$$

Equality holds if and only if

$$
\sum_{i=1}^{n_{1}} h_{i i}^{n+1}=\sum_{t=n_{1}+1}^{n} h_{t t}^{n+1} .
$$

Using again the Gauss equation, we find that

$$
\begin{gathered}
n_{2} \frac{\Delta_{1} f_{1}}{f_{1}}+n_{1} \frac{\Delta_{2} f_{2}}{f_{2}}=\tau-\sum_{1 \leq j<k \leq n_{1}} K\left(e_{j} \wedge e_{k}\right)-\sum_{n_{1}+1 \leq s<t \leq n} K\left(e_{s} \wedge e_{t}\right)= \\
=\tau-\frac{n_{1}\left(n_{1}-1\right)\left(c-3 u^{2}\right)}{8}-\sum_{1 \leq j<k \leq n_{1}} g^{2}\left(P e_{j}, e_{k}\right) \frac{3\left(c+u^{2}\right)}{4}+ \\
+\left(\frac{c+u^{2}}{4}+u^{\prime}\right)\left(n_{1}-1\right)-\sum_{r=n+1}^{2 m+1} \sum_{1 \leq j<k \leq n_{1}}\left(h_{j j}^{r} h_{k k}^{r}-\left(h_{j k}^{r}\right)^{2}\right)- \\
-\frac{n_{2}\left(n_{2}-1\right)\left(c-3 u^{2}\right)}{8}-\sum_{n_{1}+1 \leq s<t \leq n} g^{2}\left(P e_{s}, e_{t}\right) \frac{3\left(c+u^{2}\right)}{4}- \\
-\sum_{r=n+1}^{2 m+1} \sum_{n_{1}+1 \leq s<t \leq n}\left(h_{s s}^{r} h_{t t}^{r}-\left(h_{s t}^{r}\right)^{2}\right) .
\end{gathered}
$$

Combining (3.8) and (3.10) and taking account of (3.4), we obtain

$$
\begin{aligned}
& n_{2} \frac{\Delta_{1} f_{1}}{f_{1}}+n_{1} \frac{\Delta_{2} f_{2}}{f_{2}} \leq \tau-\frac{n(n-1)\left(c-3 u^{2}\right)}{8}+n_{1} n_{2} \frac{c-3 u^{2}}{4}- \\
&- \frac{\delta}{2}+\left(\frac{c+u^{2}}{4}+u^{\prime}\right)\left(n_{1}-1\right)- \\
&-\sum_{1 \leq j<k \leq n_{1}} g^{2}\left(P e_{j}, e_{k}\right) \frac{3\left(c+u^{2}\right)}{4}-\sum_{n_{1}+1 \leq s<t \leq n} g^{2}\left(P e_{s}, e_{t}\right) \frac{3\left(c+u^{2}\right)}{4} .
\end{aligned}
$$

Hence, from (3.6), the inequality (3.11) reduces to

$$
\begin{aligned}
n_{2} \frac{\Delta_{1} f_{1}}{f_{1}}+ & n_{1} \frac{\Delta_{2} f_{2}}{f_{2}} \leq \frac{n^{2}}{4}\|H\|^{2}+n_{1} n_{2} \frac{c-3 u^{2}}{4}-\left(\frac{c+u^{2}}{4}+u^{\prime}\right) n_{2}+ \\
& +\frac{3\left(c+u^{2}\right)}{4} \sum_{n_{1}+1 \leq j<t \leq n} g^{2}\left(P e_{j}, e_{t}\right) \leq \\
\leq & \frac{n^{2}}{4}\|H\|^{2}+n_{1} n_{2} \frac{c-3 u^{2}}{4}-\left(\frac{c+u^{2}}{4}+u^{\prime}\right) n_{2}+
\end{aligned}
$$




$$
+\frac{3\left(c+u^{2}\right)}{4} \min \left\{n_{1}, n_{2}\right\}
$$

We distinguish two cases:

(a) $n_{1} \leq n_{2}$, in this case the inequality (3.12) implies (3.1).

(b) $n_{1}>n_{2}$, in this case the inequality (3.12) also becomes (3.1). It completes the proof.

Remark 3.1. If $f_{2} \equiv 1$, then the inequality (3.1) is exactly the inequality (6) from [21] for warped products.

Corollary 3.1. Let $x$ be an isometric immersion of an n-dimensional totally real doubly warped product ${ }_{f_{2}} M_{1} \times_{f_{1}} M_{2}$ into a $(2 m+1)$-dimensional locally conformal almost cosymplectic manifold of pointwise constant $\varphi$-sectional curvature $c$ whose structure vector field $\xi$ is tangent to $M_{1}$. Then, we have:

$$
n_{2} \frac{\Delta_{1} f_{1}}{f_{1}}+n_{1} \frac{\Delta_{2} f_{2}}{f_{2}} \leq \frac{n^{2}}{4}\|H\|^{2}+n_{1} n_{2} \frac{c-3 u^{2}}{4}-\left(\frac{c+u^{2}}{4}+u^{\prime}\right) n_{2},
$$

where $n_{i}=\operatorname{dim} M_{i}$ and $\Delta_{i}$ is the Laplacian operator of $M_{i}, i=1,2$.

Moreover, the equality case of (3.13) holds if and only if $x$ is a mixed totally geodesic immersion and $n_{1} H_{1}=n_{2} H_{2}$, where $H_{i}, i=1,2$, are the partial mean curvature vectors.

Proof. Let $f_{2} M_{1} \times{ }_{f_{1}} M_{2}$ be a doubly warped product submanifold of a locally conformal almost cosymplectic manifold $\widetilde{M}(c)$. Then we have $g\left(P e_{i}, e_{s}\right)=0$ for $0 \leq i \leq n_{1}, n_{1}+1 \leq s \leq n$. Therefore, by (3.12) we can easily obtain the inequality (3.13). Also, we see that the equality of (3.12) holds if and only if

$$
h_{j t}^{r}=0,1 \leq j \leq n_{1}, n_{1}+1 \leq t \leq n, n+1 \leq r \leq 2 m+1,
$$

and

$$
\sum_{i=1}^{n_{1}} h_{i i}^{r}=\sum_{t=n_{1}+1}^{n} h_{t t}^{r}=0, n+2 \leq r \leq 2 m+1 .
$$

Obviously (3.14) is equivalent to the mixed totally geodesic of the doubly warped product ${ }_{f_{2}} M_{1} \times_{f_{1}} M_{2}$ and (3.9) and (3.15) imply $n_{1} H_{1}=n_{2} H_{2}$. The converse statement is straightforward.

As applications, we obtain certain obstructions to the existence of minimal totally real doubly warped product submanifolds in locally conformal almost cosymplectic manifolds.

By using the above corollary, we can obtain some important consequences:

Corollary 3.2. Let ${ }_{f_{2}} M_{1} \times_{f_{1}} M_{2}$ be a totally real doubly warped product in a $(2 m+1)$-dimensional locally conformal almost cosymplectic manifold of pointwise constant $\varphi$-sectional curvature $c$ whose structure vector field $\xi$ is tangent to $M_{1}$. If the warping functions are harmonic, then ${ }_{f_{2}} M_{1} \times_{f_{1}} M_{2}$ admits no minimal totally real immersion into a locally conformal almost cosymplectic manifold $\widetilde{M}(c)$ with $c<\frac{1}{n_{1}-1}\left(u^{2}+3 n_{1} u^{2}+4 u^{\prime}\right)$.

Proof. Assume $f_{1}$ is a harmonic function on $M_{1}, f_{2}$ is a harmonic function on $M_{2}$ and ${ }_{f_{2}} M_{1} \times_{f_{1}} M_{2}$ admits a minimal totally real immersion in a locally conformal almost cosymplectic manifold $\widetilde{M}(c)$. Then, the inequality (3.13) becomes $c \geq \frac{1}{n_{1}-1}\left(u^{2}+3 n_{1} u^{2}+4 u^{\prime}\right)$.

Corollary 3.3. Let ${ }_{f_{2}} M_{1} \times_{f_{1}} M_{2}$ be a totally real doubly warped product in a $(2 m+1)$-dimensional locally conformal almost cosymplectic manifold of pointwise constant $\varphi$-sectional curvature c whose structure vector field $\xi$ is tangent to $M_{1}$. If the warping functions $f_{1}$ and $f_{2}$ of $f_{2} M_{1} \times_{f_{1}} M_{2}$ are eigenfunctions of the Laplacian on $M_{1}$ and $M_{2}$, respectively, with corresponding eigenvalues $\lambda_{1}>0$ and $\lambda_{2}>0$, respectively, then ${ }_{f_{2}} M_{1} \times_{f_{1}} M_{2}$ admits no minimal totally real immersion into a locally conformal almost cosymplectic manifold $\widetilde{M}(c)$ with $c \leq \frac{1}{n_{1}-1}\left(u^{2}+3 n_{1} u^{2}+4 u^{\prime}\right)$.

Corollary 3.4. Let ${ }_{f_{2}} M_{1} \times_{f_{1}} M_{2}$ be a totally real doubly warped product in a $(2 m+1)$-dimensional locally conformal almost cosymplectic manifold of pointwise constant $\varphi$-sectional curvature $c$ whose structure vector field $\xi$ is tangent to $M_{1}$. If one of the warping functions is harmonic and the other one is an eigenfunction of the Laplacian with corresponding eigenvalue $\lambda>0$, then ${ }_{f_{2}} M_{1} \times_{f_{1}} M_{2}$ admits no minimal totally real immersion into a locally conformal almost cosymplectic manifold $\widetilde{M}(c)$ with $c \leq \frac{1}{n_{1}-1}\left(u^{2}+3 n_{1} u^{2}+4 u^{\prime}\right)$. 
Theorem 3.2. Let $x$ be an isometric immersion of an n-dimensional doubly warped product ${ }_{f_{2}} M_{1} \times_{f_{1}} M_{2}$ into a $(2 m+1)$-dimensional locally conformal almost cosymplectic manifold of pointwise constant $\varphi$-sectional curvature $c$ whose structure vector field $\xi$ is tangent to $M_{2}$. Then:

$$
n_{2} \frac{\Delta_{1} f_{1}}{f_{1}}+n_{1} \frac{\Delta_{2} f_{2}}{f_{2}} \leq \frac{n^{2}}{4}\|H\|^{2}+n_{1} n_{2} \frac{c-3 u^{2}}{4}-\left(\frac{c+u^{2}}{4}+u^{\prime}\right) n_{1}+\frac{3\left(c+u^{2}\right)}{4} n_{2},
$$

where $n_{i}=\operatorname{dim} M_{i}$ and $\Delta_{i}$ is the Laplacian operator of $M_{i}, i=1,2$.

Remark 3.2. If $f_{2} \equiv 1$, then the inequality (3.16) is exactly the inequality from Theorem 8 from [21] for warped products.

Corollary 3.5. Let $x$ be an isometric immersion of an n-dimensional totally real doubly warped product ${ }_{f_{2}} M_{1} \times_{f_{1}} M_{2}$ into a $(2 m+1)$-dimensional locally conformal almost cosymplectic manifold of pointwise constant $\varphi$-sectional curvature $c$ whose structure vector field $\xi$ is tangent to $M_{2}$. Then, we have:

$$
n_{2} \frac{\Delta_{1} f_{1}}{f_{1}}+n_{1} \frac{\Delta_{2} f_{2}}{f_{2}} \leq \frac{n^{2}}{4}\|H\|^{2}+n_{1} n_{2} \frac{c-3 u^{2}}{4}-\left(\frac{c+u^{2}}{4}+u^{\prime}\right) n_{1},
$$

where $n_{i}=\operatorname{dim} M_{i}$ and $\Delta_{i}$ is the Laplacian operator of $M_{i}, i=1,2$.

Moreover, the equality case of (3.17) holds if and only if $x$ is a mixed totally geodesic immersion and $n_{1} H_{1}=n_{2} H_{2}$, where $H_{i}, i=1,2$, are the partial mean curvature vectors.

Corollary 3.6. Let ${ }_{f_{2}} M_{1} \times{ }_{f_{1}} M_{2}$ be a totally real doubly warped product in a $(2 m+1)$-dimensional locally conformal almost cosymplectic manifold of pointwise constant $\varphi$-sectional curvature $c$ whose structure vector field $\xi$ is tangent to $M_{2}$. If the warping functions are harmonic, then ${ }_{f_{2}} M_{1} \times_{f_{1}} M_{2}$ admits no minimal totally real immersion into a locally conformal almost cosymplectic manifold $\widetilde{M}(c)$ with $c<\frac{1}{n_{2}-1}\left(u^{2}+3 n_{2} u^{2}+4 u^{\prime}\right)$.

Corollary 3.7. Let ${ }_{f_{2}} M_{1} \times_{f_{1}} M_{2}$ be a totally real doubly warped product in a $(2 m+1)$-dimensional locally conformal almost cosymplectic manifold of pointwise constant $\varphi$-sectional curvature $c$ whose structure vector field $\xi$ is tangent to $M_{2}$. If the warping functions $f_{1}$ and $f_{2}$ of $f_{2} M_{1} \times_{f_{1}} M_{2}$ are eigenfunctions of the Laplacian on $M_{1}$ and $M_{2}$, respectively, with corresponding eigenvalues $\lambda_{1}>0$ and $\lambda_{2}>0$, respectively, then $f_{f_{2}} M_{1} \times_{f_{1}} M_{2}$ admits no minimal totally real immersion into a locally conformal almost cosymplectic manifold $\widetilde{M}(c)$ with $c \leq \frac{1}{n_{2}-1}\left(u^{2}+3 n_{2} u^{2}+4 u^{\prime}\right)$.

Corollary 3.8. Let ${ }_{f_{2}} M_{1} \times_{f_{1}} M_{2}$ be a totally real doubly warped product in a $(2 m+1)$-dimensional locally conformal almost cosymplectic manifold of pointwise constant $\varphi$-sectional curvature $c$ whose structure vector field $\xi$ is tangent to $M_{2}$. If one of the warping functions is harmonic and the other one is an eigenfunction of the Laplacian with corresponding eigenvalue $\lambda>0$, then ${ }_{f_{2}} M_{1} \times_{f_{1}} M_{2}$ admits no minimal totally real immersion into a locally conformal almost cosymplectic manifold $\widetilde{M}(c)$ with $c \leq \frac{1}{n_{2}-1}\left(u^{2}+3 n_{2} u^{2}+4 u^{\prime}\right)$.

\section{References}

[1] Bishop, R. L. and O'Neill, B., Manifolds of negative curvature. Trans. Amer. Math. Soc. 145 (1969), 1-49.

[2] Blair, D. E., Contact Manifolds in Riemannian Geometry. Lecture Notes in Math. 509, Springer, Berlin, 1976.

[3] Chen, B. Y., Some pinching and classification theorems for minimal submanifolds. Arch. Math. 60 (1993), 568-578.

[4] Chen, B. Y., On isometric minimal immersions from warped products into real space forms. Proc. Edinburgh Math. Soc. 45 (2002), $579-587$.

[5] Chen, B. Y. and Dillen, F., Optimal inequalities for multiply warped product submanifolds. Int. Electron. J. Geom., Vol. 1 (2008), 1-11.

[6] Crasmareanu, M., Adapted metrics and Webster curvature on three classes of 3-dimensional geometries. Int. Electron. J. Geom., 7 (2) (2014), 37-46.

[7] Malek, F. and Nejadakbary, V., Warped product submanifold in generalized Sasakian space form. Acta Math. Acad. Paedagog. Nyhazi. (N.S.) 27 no. 2 (2011), 325-338.

[8] Matsumoto, K. and Mihai, I., Warped product submanifolds in Sasakian space forms. SUT Journal of Mathematics 38 (2002), 135-144.

[9] Matsumoto, K., Mihai, I. and Rosca, R., A certain locally conformal almost cosymplectic manifold and its submanifolds. Tensor N.S. 51 (1) (1992), 91-102.

[10] Mihai, A., Warped product submanifolds in complex space forms. Acta Sci. Math. (Szeged) 70 (2004), 419-427.

[11] Mihai, A., Warped product submanifolds in quaternion space forms. Rev. Roumaine Math. Pures Appl. 50 (2005), 283-291.

[12] Mihai, A., Mihai I. and Miron, R. (Eds.), Topics in Differential Geometry, Ed. Academiei Romane, Bucuresti, 2008.

[13] Mihai, I. and Presura, I., An improved Chen first inequality for Legendrian submanifolds in Sasakian space forms. Period. Math. Hung. 74 (2) (2017), 220-226.

[14] Murathan, C., Arslan, K., Ezentas, R. and Mihai, I., Warped product submanifolds in Kenmotsu space forms. Taiwanese J. Math. 10 (2006), 1431-1441. 
[15] Olszak, Z., Locally conformal almost cosymplectic manifolds. Collq. Math. 57(1) (1989), 73-87.

[16] Olteanu, A., A general inequality for doubly warped product submanifolds. Math. J. Okayama Univ. 52 (2010), 133-142.

[17] Olteanu, A., Recent results in the geometry of warped product submanifolds, Matrix Rom, 2011.

[18] Olteanu, A., Doubly warped product submanifolds in generalized Sasakian space forms, Proceedings RIGA 2014, Ed. Univ. Bucuresti (2014), 174-184.

[19] Olteanu, A., Doubly warped products in S-space forms. Rom. J. Math. Comput. Sci. 4 Issue 1 (2014), 111-124.

[20] Ünal, B., Doubly warped products. Differ. Geom. App. 15(3) (2001), 253-263.

[21] Yoon, D. W., Cho, K. S. and Han, S. G., Some inequalities for warped products in locally conformal almost cosymplectic manifolds. Note Mat. 23 (1) (2004), 51-60.

\section{Affiliations}

\section{ANDREEA OLTEANU}

AdDRESS: University of Agronomic Sciences and Veterinary Medicine of Bucharest, Dept. of Mathematics, Physics and Terrestrial Measurements, 011464, Bucharest-Romania.

E-MAIL: andreea_d_olteanu@yahoo.com

ORCID ID : orcid.org/0000-0002-2841-2029 